\title{
Stability analysis of a pneumatically actuated inverted pendulum
}

\author{
Federico Colombo, Luigi Mazza, Giuseppe Pepe, Terenziano Raparelli, Andrea Trivella \\ Politecnico di Torino, Technical University, \\ C.so Duca degli Abruzzi 24, 10129, Torino, Italy
}

\begin{abstract}
This paper deals with the stabilization of an inverted pendulum on cart; the latter is pneumatically actuated by a double acting cylinder controlled by low cost proportional valves. In particular, a numerical model of whole system is developed in order to find the ability of the pneumatic actuation in stabilizing the pendulum and evaluate its bandwidth. A cascade of two control loops (the inner one for the pendulum angle, the outer one for the cart displacement) are analyzed and proper compensators are defined. The possibility of introducing an additional loop to control the force exerted by the actuator on the cart is evaluated.
\end{abstract}

\section{Introduction}

Majority of the inverted pendulum based applications belong to the field of human transportation such as self-balancing scooters [1], unicycles [2] or electric motorbikes [3]; recently many solutions have grown in the version of "autonomous robots" [4-6]. The inverted pendulum still remains an interesting system for didactic use or to test new control types; to this aim papers focused on different architectures and control strategies $[7,8]$ can be found. Moreover, applications in the field of biomechanics are quite interesting and challenging; in particular, the gait modelling and stability of biped robots [9] and postural exoskeleton balancing [10] are based on inverted pendulum models. Most of the applications make use of electric actuators, appreciated for their high bandwidth. Anyway, some others make use of pneumatic technology [11-14], which presents some advantages such as the low cost and bandwidth sufficient to obtain stabilization. In [11] a low cost pneumatic actuator is used to control the system, together with potentiometers that measure the linear and angular displacements of cart and pendulum respectively. The control is implemented on a notebook equipped with a PCMCIA card. In [12] an inner loop controlling the actuating force of a pneumatic cylinder was studied, while position and angle were controlled in an outer loop. A friction estimator offsets the control signal for the force control to compensate for friction. However the work is purely numerical.

The authors have already designed and manufactured a prototype of inverted pendulum mounted on cart and pneumatically actuated. The architecture of the system is described [13, 14]. Four proportional valves were used to supply the double-acting cylinder and 
potentiometers are employed to feedback the displacement signals; in particular, in [14] a common industrial logic controller (PLC) was used to control the pneumatic servosystem.

In this paper, the numerical model of the pneumatic system is detailed and various compensators are designed in order to achieve good stability margins and bandwidth. A cascade of two control loops (the inner one for the pendulum angle, the outer one for the cart displacement) is analysed and stability is discussed. The possibility of introducing an additional inner loop (third loop) to control the force exerted by the pneumatic cylinder is also evaluated and discussed.

\section{Mathematical model}

The inverted pendulum on cart is described by equations (1), (2) which represent the rotational and translational equilibrium:

$$
\begin{gathered}
g \sin \vartheta-l \ddot{\vartheta}+\ddot{x} \cos \vartheta=0 \\
\left(m_{p}+m_{c}\right) \ddot{x}+m_{p} l \dot{\vartheta}^{2} \sin \vartheta-m_{p} l \ddot{\vartheta} \cos \vartheta+b_{c} \dot{x}-F=0
\end{gathered}
$$

After linearization of these equations, it is possible to obtain the transfer function between angle $\theta$ and cart position $x$

$$
\frac{x(s)}{\theta(s)}=G_{x / \theta}=\frac{l s^{2}-g}{s^{2}}
$$

and the transfer function between the force $F$ applied by the cylinder to the cart and angle $\theta$ :

$$
\frac{\vartheta(s)}{F(s)}=G_{\theta / F}=\frac{s}{m_{c} l s^{3}+b_{c} l s^{2}-g\left(m_{p}+m_{c}\right) s-b_{c} g}
$$

The pneumatic cylinder is modelled considering the equilibrium equation (5) and the mass balance equations $(6,7)$ for the two chambers (rear chamber $\mathrm{A}$ and front chamber B). Friction force in pneumatic actuators depends on many factors such as velocity, chambers pressure, greasing conditions, material and shape of the seals [15], which can lead to complex friction models. For the scope of this analysis, a classical viscous friction model was employed.

$$
\begin{gathered}
F=p_{A} A_{A}-p_{B} A_{B}-p_{a}\left(A_{A}-A_{B}\right)-b_{p} \dot{x}-M_{p} \ddot{x} \\
\dot{m}_{A}=\frac{\dot{p}_{A} \rho_{0}}{p_{0}}\left(V_{A 0}+A_{A} x\right)+\frac{p_{A} \rho_{0}}{p_{0}} A_{A} \dot{x} \\
\dot{m}_{B}=\frac{\dot{p}_{B} \rho_{0}}{p_{0}}\left(V_{B 0}-A_{B} x\right)-\frac{p_{B} \rho_{0}}{p_{0}} A_{B} \dot{x}
\end{gathered}
$$

where $\rho_{0}$ and $p_{0}$ are the air density and pressure in reference conditions.

The air flow through the valves is given by

$$
\dot{m}=\rho_{0} C k_{T} \frac{\Delta p}{(1-b)}
$$


where $k_{T}=\sqrt{T / T_{0}}$ is the temperature ratio, $b$ the critical ratio and $C$ the valve conductance, $\Delta p$ is the pressure difference between chambers. The pressure difference on the supply valve is $\Delta p=p_{\mathrm{s}}-p$ and $\Delta p=p-p_{\mathrm{a}}$ on the discharge valve, where $p_{\mathrm{s}}$ is the supply pressure and $p_{\mathrm{a}}$ is the ambient pressure, while $p$ is the pressure in chambers.

The proportional valves model is linearized around the operating point defined by $\left(p_{\mathrm{s}}+p_{\mathrm{a}}\right) / 2$. Neglecting the opening time of the valve, this expression results to be proportional to the command signal $u$ to the valve:

$$
\dot{m}=K u \Delta p
$$

Input signal $u$ can change in range $-1<u<1$; positive/negative values refer to outward/backward cylinder motion.

From relations (3) to (9) it is possible to obtain the transfer function $G_{\theta / u}=\frac{\theta(s)}{u(s)}$ between input signal $u$ and angle $\theta$.

$$
\begin{aligned}
& \quad G_{\theta / u}=\frac{\theta(s)}{u(s)}= \\
& \frac{\left(K_{A} A_{A}+K_{B} A_{B}\right) s}{\left(M_{p}+m_{c}\right) l s^{4}+\left(b_{c}+b_{p}\right) l s^{3}+\left(C_{A} A_{A}+C_{B} A_{B}\right) l s^{2}-\left(M_{p}+m_{c}+m_{p}\right) g s^{2}-\left(b_{c}+b_{p}\right) g s-\left(C_{A} A_{A}+C_{B} A_{B}\right) g}
\end{aligned}
$$

Moreover, the relationship between the force $F$ available in output from actuator and variables $u$ and $x$ is made explicit, where the cart position is assimilated to a disturbance variable and $u$ is the input variable:

$$
F(s)=\frac{K_{A} A_{A}+K_{B} A_{B}}{s} u(s)-\left(M_{p} s^{2}+b_{p} s+C_{A} A_{A}+C_{B} A_{B}\right) x(s)
$$

where

$$
\begin{aligned}
K_{A} & =p_{0} \frac{K \frac{p_{s}}{2}}{\rho_{0} V_{A 0}} \\
K_{B} & =p_{0} \frac{K \frac{p_{s}}{2}}{\rho_{0} V_{B 0}} \\
K & =\rho_{0} C k_{T} \\
C_{A} & =\frac{\frac{p_{S}}{2}}{V_{A 0}} A_{A} \\
C_{B} & =\frac{\frac{p_{S}}{2}}{V_{B 0}} A_{B}
\end{aligned}
$$

\section{Control architecture}

The control is aimed at controlling the pendulum angle and the cart position in the set point reducing to zero the error between set and feedback. The control on $x$ is necessary as the cylinder stroke is limited to $\pm 250 \mathrm{~mm}$. Figure 1 outlines the block diagram of the control 
architecture, which consists of a cascade of two loops to control angle $\theta$ (inner loop) and position $x$ (outer loop).

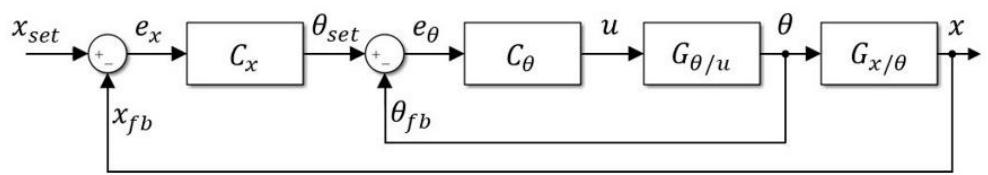

Fig. 1. Control system architecture only with $\theta$ and $x$ controlled.

As an alternative to this scheme, a further loop is introduced to control the force $F$ exerted by the cylinder on the cart. The new architecture is depicted in Figure 2. In this case, a load cell is inserted between cylinder and cart. The eventual advantages of introducing this loop are discussed here below.

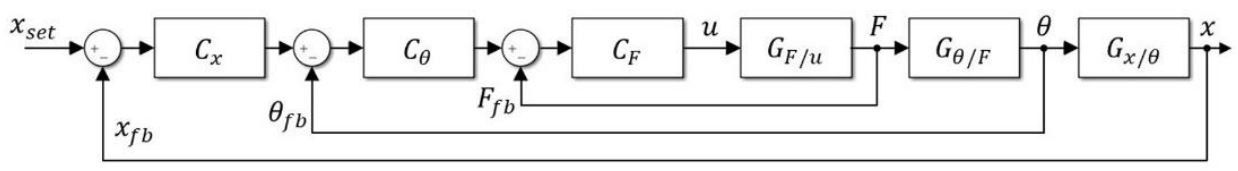

Fig. 2. Control system architecture with $F, \theta$ and $x$ controlled.

\section{The regulators}

Referring to Figures 1 and 2, transfer functions $C_{x}, C_{\theta}, C_{F}$ are the regulators of displacement, angle and force loops respectively. The regulators designed are the filtered PID (with first order filter on the derivative part) and the filtered PD. The first one assumes the following form:

$$
C_{P I D_{f}}(s)=K_{p}+\frac{K_{i}}{s}+\frac{K_{d} s}{\tau_{d} s+1}
$$

where $K_{p}, K_{i}, K_{d}$ are the proportional, integral and derivative gains, while $\tau_{d}$ is the filter time constant. The second one lacks of the integral part and is equivalent to a phase lead compensator.

For the angle loop, compensator $C_{\theta}$ was found to be of PIDf type. For the position loop, two alternatives compensators $C_{x}$ were designed: a PIDf and a PDf.

\section{Results and discussion}

A stability analysis of the linearized system is performed around the operating point, characterized by $x=0, \theta=0$.

The compensators of the control architecture of Figure 1 were designed with Matlab pid Tuner toolbox considering the following constraints: a) the crossover frequency of the inner angle loop was fixed to $10 \mathrm{rad} / \mathrm{s}$; b) the crossover frequency of the displacement outer loop 
was fixed to $1 \mathrm{rad} / \mathrm{s}$; c) the minimum phase margin of both loops was set to $50^{\circ}$. Stability was verified both using the Nyquist criterion and analysing the sign of the closed loop transfer function roots.

\subsection{Control architecture 1: angle $\theta$ and displacement $x$}

Table 1. Compensators of $x$ and $\theta$ loops

\begin{tabular}{|c|c|c|c|c|c|c|c|}
\hline Compensator & type & $K_{p}$ & $K_{i}$ & $K_{d}$ & $\tau_{d}$ & $\begin{array}{c}\text { Gain } \\
\text { Margin }\end{array}$ & $\begin{array}{c}\text { Phase } \\
\text { Margin }\end{array}$ \\
\hline$C_{\theta}$ & PIDf & 2.08 & 9.87 & 0.109 & 0.000875 & $\begin{array}{c}5.33 \mathrm{~dB} \\
\text { (a) } 17.6 \mathrm{rad} / \mathrm{s}\end{array}$ & $\begin{array}{c}50^{\circ} \\
\text { (a) } 10 \mathrm{rad} / \mathrm{s}\end{array}$ \\
\hline$C_{x}$ & PDf & 0.00702 & - & 0.0467 & 0.285 & $\begin{array}{c}10.9 \mathrm{~dB} \\
@ 3.59 \mathrm{rad} / \mathrm{s}\end{array}$ & $\begin{array}{c}55.8^{\circ} \\
@ 1 \mathrm{rad} / \mathrm{s}\end{array}$ \\
\hline$C_{x}$ & PIDf & $\begin{array}{c}- \\
0.00868\end{array}$ & $\begin{array}{c}- \\
0.000401\end{array}$ & $\begin{array}{c}- \\
0.0461\end{array}$ & 0.183 & $\begin{array}{c}11.1 \mathrm{~dB} \\
@ 4.31 \mathrm{rad} / \mathrm{s}\end{array}$ & $\begin{array}{c}59.1^{\circ} \\
@ 1 \mathrm{rad} / \mathrm{s}\end{array}$ \\
\hline
\end{tabular}

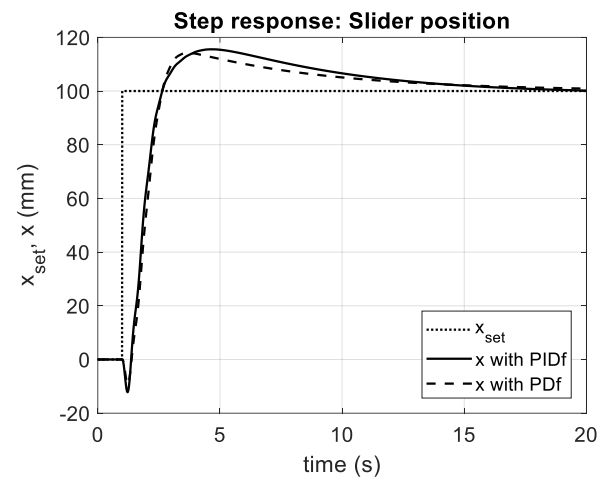

(a)

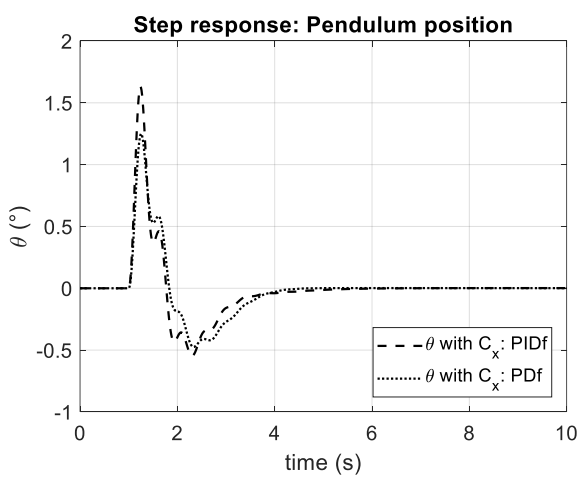

(b)

Fig. 3. Response to a step displacement with two different compensators $C_{x}$, (a) cart position and (b) pendulum angle.

The performance of the controlled system is evaluated with the response to a displacement step. Figures $3 \mathrm{a}-3 \mathrm{~b}$ show the cart displacement and the pendulum angle after a $100 \mathrm{~mm}$ step, considering the designed compensators $C_{x}$.

The settling time, evaluated to reach $2 \%$ of the static value, is $13.9 \mathrm{~s}$ and $13.6 \mathrm{~s}$ for the PDf and the PIDf respectively, while the overshoot is $14.2 \%$ and $15.5 \%$ respectively. In both cases the angular oscillations is limited in range $\pm 2^{\circ}$, see Figure $3 \mathrm{~b}$.

\subsection{Control architecture 2: force $\mathrm{F}$, angle $\boldsymbol{\theta}$ and displacement $\boldsymbol{x}$}

The force control is based on eq. (11), which describes the dependency on $x$, assimilated to a disturbance, and input signal $\mathrm{u}$. The block diagram of Figure 4 depicts the force loop which have been considered to design compensator $C_{F}$.

Eq. (13) defines the disturbance transfer function between $x$ and $\mathrm{F}$, while eq. (14) expresses it in the normalized form. 


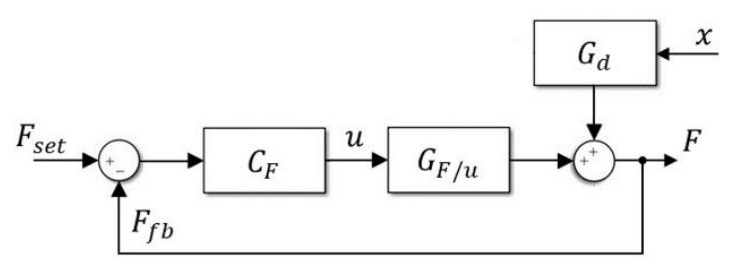

Fig 4. Control loop of the controlled variable $F$.

$$
\begin{gathered}
G_{F / u}(s)=\frac{K_{A} A_{A}+K_{B} A_{B}}{s}=\frac{G_{1}}{s} \\
G_{d}(s)=-\left(M_{P} s^{2}+b_{P} s+C_{A} A_{A}+C_{B} A_{B}\right) \\
G_{d}(s)=-\left(C_{A} A_{A}+C_{B} A_{B}\right)\left(\frac{M_{P} s^{2}}{C_{A} A_{A}+C_{B} A_{B}}+\frac{b_{P} s}{C_{A} A_{A}+C_{B} A_{B}}+1\right)= \\
=G_{2 c}\left(\frac{s^{2}}{\sigma_{d}^{2}}+2 \zeta_{d} \frac{s}{\sigma_{d}}+1\right)=G_{2 c} G_{2}(s)
\end{gathered}
$$

where $\sigma_{d} \cong 33,5 \mathrm{rad} / \mathrm{s} \zeta_{d} \cong 0,63$. These parameters are only function of physical parameters of the system.

The closed loop transfer function is

$$
F(s)=\frac{1}{\frac{1}{C_{F} G_{1}} s+1} F_{s e t}+\frac{\frac{G_{d}}{C_{F} G_{1}} s}{\frac{1}{C_{F} G_{1}} s+1} x
$$

while the open loop transfer function (see Figure 4) is

$$
C_{F} G_{F / u}=\frac{C_{F} G_{1}}{S}
$$

The system stability is assured as the phase is always beyond $-90^{\circ}$. Considering constant Fset, the variation of $\mathrm{F}$ due to a variation of disturbance $\mathrm{x}$ is expressed by

$$
F(s)=\frac{\frac{G_{d}}{C_{F} G_{1}} s}{\frac{1}{C_{F} G_{1}} s+1} x
$$

while the disturbance stiffness is defined as the inverse function:

$$
\frac{x}{F}(s)=\frac{1+C_{F} G_{F / u}}{G_{d}}=\frac{C_{F} G_{1} / G_{2 c}}{s} \frac{\left(\frac{1}{C_{F} G_{1}} s+1\right)}{G_{2}(s)}
$$


It is important to have a high disturbance stiffness in order to have a negligible influence of the disturbance on the controlled variable. Figure 5 shows the Bode diagram of the disturbance stiffness: introducing the controller, it is clear that it remains high in a wide frequency range, sufficient to cover the frequency of variation of displacement $\mathrm{x}$.

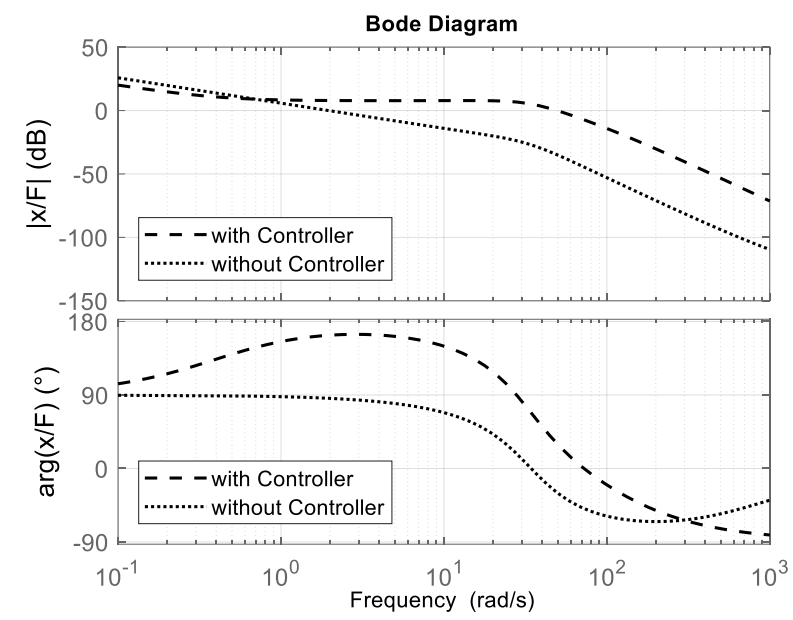

Fig. 5. Bode diagram of disturbance stiffness, with and without compensator $C_{F}$.

The adopted compensator is expressed by

$$
C_{F}(s)=K_{p}+\frac{K_{d} s}{\tau_{d} s+1}=0.5+\frac{1.24 s}{0.01 s+1}
$$

It gives wide stability margins and a crossover frequency higher than $100 \mathrm{rad} / \mathrm{s}$. Compensators $C_{\theta}$ e $C_{x}$ are also reported in Table 2.

Table 2. Compensators of $\mathrm{x}$ and $\theta$ loops in case of force control

\begin{tabular}{|c|c|c|c|c|c|c|c|}
\hline \multicolumn{2}{|c|}{ Compensator } & \multirow{2}{*}{$\begin{array}{l}\text { type } \\
88.8\end{array}$} & \multirow{2}{*}{$\begin{array}{r}\boldsymbol{K}_{\boldsymbol{p}} \\
218\end{array}$} & \multirow{2}{*}{$\begin{aligned} \boldsymbol{K}_{\boldsymbol{i}} \\
8.99\end{aligned}$} & \multirow{2}{*}{$\begin{array}{ll}\boldsymbol{K}_{\boldsymbol{d}} \quad \boldsymbol{\tau}_{\boldsymbol{d}} \\
0.000875\end{array}$} & \multirow{2}{*}{$\begin{array}{c}\begin{array}{c}\text { Gain } \\
\text { Margin }\end{array} \\
-11.9 \mathrm{~dB} \\
@ 1.76 \mathrm{rad} / \mathrm{s}\end{array}$} & \multirow{2}{*}{$\begin{array}{c}\begin{array}{c}\text { Phase } \\
\text { Margin }\end{array} \\
50^{\circ} \\
\text { a } 10 \mathrm{rad} / \mathrm{s}\end{array}$} \\
\hline$C_{\theta}$ & PIDf & & & & & & \\
\hline$C_{x}$ & PDf & 0.0149 & - & -0.074 & 0.267 & $\begin{array}{c}9.35 \mathrm{~dB} \\
@ 5.77 \mathrm{rad} / \mathrm{s}\end{array}$ & $\begin{array}{c}64.9^{\circ} \\
\text { (a) } 1 \mathrm{rad} / \mathrm{s}\end{array}$ \\
\hline$C_{x}$ & PIDf & $\begin{array}{c}- \\
0.0159\end{array}$ & -0.000805 & $\begin{array}{c}- \\
0.0751\end{array}$ & 0.369 & $\begin{array}{c}10.7 \mathrm{~dB} \\
@, 5.25 \mathrm{rad} / \mathrm{s}\end{array}$ & $\begin{array}{c}59^{\circ} \\
@ 1 \mathrm{rad} / \mathrm{s}\end{array}$ \\
\hline
\end{tabular}

Figure 6 shows the response to a $100 \mathrm{~mm}$ displacement step in terms of cart position and pendulum angle obtained with two different compensators $C_{x}$. 


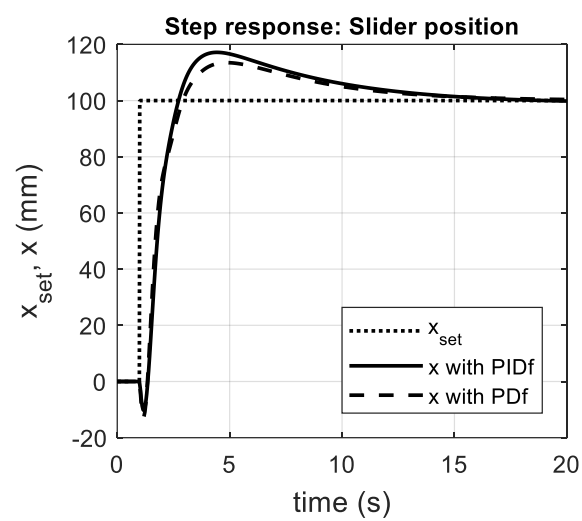

(a)

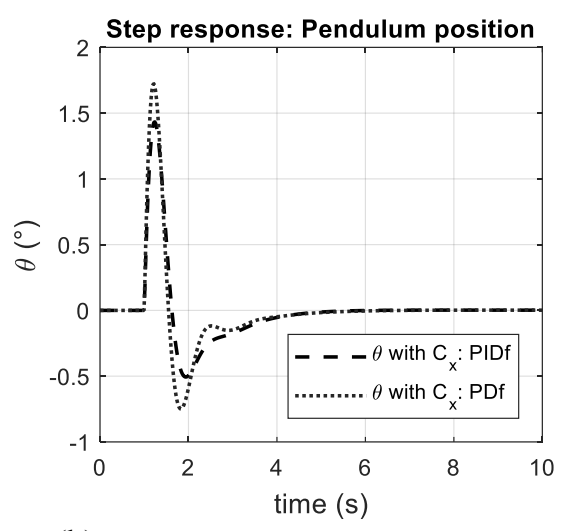

(b)

Fig. 6. Step response with two different compensators $C_{x}$, (a) slider position and (b) pendulum position.

The settling time, evaluated to reach $2 \%$ of the static value, is $12.1 \mathrm{~s}$ and $12.7 \mathrm{~s}$ for the PDf and the PIDf compensators respectively, while the overshoot is $13.5 \%$ and $17.2 \%$ respectively. In both cases the angular oscillations is limited in range $\pm 2^{\circ}$, see Figure $6 \mathrm{~b}$.

\section{Comparison}

In both control architectures the two proposed sets of compensators don't really differ. In architecture 2 (with the force loop) the settling time is a little smaller $(-6,6 \%)$ considering the same type of compensator $C_{x}$.

Nevertheless, to have a more complete analysis of the comparison between the two architectures, a non-linear control system should be considered and experimental tests should be carried out.

\section{Conclusions}

Control architectures to stabilize a pneumatically actuated inverted pendulum were described in this paper and proper PD and PID compensators are designed. Cascade controls with two or three loops were considered to control the actuator force, the pendulum angle and the displacement. Both architectures allow the pendulum to be stable. In particular, the use of an additional internal force loop seems to be not so favorable in terms of transient response (settling time) compared to the cost needed for the force transducer. In order to further investigate and compare these solutions, a non-linear analysis will be carried out together with experimental tests.

\section{Nomenclature}

$$
\begin{array}{ll}
x: & \text { cart position }(\mathrm{m}) \\
\theta: & \text { pendulum angle }(\mathrm{rad}) \\
A_{A}, A_{B}: & \text { cylinder sections }\left(\mathrm{m}^{2}\right) \\
C: & \text { valve conductance }\left(\frac{\mathrm{m}^{3}}{\mathrm{sPa}}\right)_{A N R} \\
K: & \text { valve linearization coefficient }(\mathrm{kg} / \mathrm{sPa})
\end{array}
$$


$M_{p}: \quad$ cylinder rod mass $(\mathrm{kg})$

$T_{0}$ : $\quad$ temperature in reference conditions $(K)$

$V_{A 0}, V_{B 0}$ : dead volumes of cylinder $\left(\mathrm{m}^{3}\right)$

$b_{c}$ : friction coefficient between cart and basement $(\mathrm{Ns} / \mathrm{m})$

$b_{p}$ : friction coefficient in cylinder $(\mathrm{Ns} / \mathrm{m})$

$l: \quad$ pendulum length $(m)$

$m_{c}: \quad$ cart mass $(\mathrm{kg})$

$m_{p}: \quad$ pendulum mass $(\mathrm{kg})$

$p_{0}: \quad$ reference pressure $(\mathrm{Pa})$

$p$ : $\quad$ absolute pressure $(P a)$

$p_{a}: \quad$ ambient pressure $(P a)$

$\rho_{0}: \quad$ air density in reference conditions $\left(\mathrm{kg} / \mathrm{m}^{\wedge} 3\right)$

$u$ : $\quad$ valve command signal

$\sigma_{d}: \quad$ damped natural frequency

$\zeta_{d}: \quad$ damping ratio

\section{References}

1. https://it.wikipedia.org/wiki/Segway

2. https://en.wikipedia.org/wiki/Self-balancing unicycle

3. http://litmotors.com/

4. F. Grasser, A. D'Arrigo, S. Colombi, A. C. Rufer, IEEE Transactions on Industrial Electronics, 49 (1), pp.107-114 (2002).

5. N.M. Abdul Ghani, D. Ju, H.Z. Othman, M.A. Ahmad, Proceedings on $10^{\text {th }}$ International Conference on Intelligent Systems Design and Applications, pp. 10661070, IEEE, Cairo, Egypt, (2010).

6. H.W. Kim, S. Jung, Robotica (2016); 34 (5): pp. 1186-1208.

7. J.J. Wang, Simulation Modelling Practice and Theory (19), pp. 440-449 (2011).

8. L., Wende, D. Hui, C. Kai, Proceedings of International Conference on Control 2012, pp. 190-196. IEEE, Cardiff, UK (2012).

9. S. Kajita, M. Morisawa, K. Miura, S. Nakaoka, IEEE Int. Conf. on Intelligent Robots and Systems, pp. 4489-4496, Taipei, Taiwan, (2010).

10. B. Ugurlu, C. Doppmann, M. Hamaya, P. Forni, T. Teramae, T. Noda, J. Morimoto. IEEEASME Transactions on Mechatronics, 21 (1), pp. 79-87 (2016).

11. T. Žilić, D. Pavković, D. Zorc. ISA Transactions 48 (3), pp. 327-335 (2009).

12. C. Krupke, J. Wang. International Proceedings of the Conference on Advanced Intelligent Mechatronics, pp. 812-817. IEEE, Busan, Korea (2015).

13. F. Colombo, L. Mazza, G. Pepe, T. Raparelli, A. Trivella, RAAD 2018, Mechanisms and Machine Science, 67 (2018).

14. M. Pontin, F. Colombo, L. Mazza, T. Raparelli, Proc IMechE Part I: J. Systems and Control Engineering, pp. 1-12 (2019).

15. L. Mazza, G. Belforte, ASME. J. Tribol., 139(2) 022202 (2017). 